\title{
Alternative use of oily fractions of olive oil
}

\author{
Melina Bagni* (D), Dolly Granados ${ }^{1}$ and María Reboredo² \\ IInstituto de Ingeniería Química - IIQ, Facultad de Ingeniería - FI, Universidad Nacional de San \\ Juan - UNSJ, San Juan, Argentina \\ ${ }^{2}$ Departamento de Ingeniería de Materiales, Instituto de Investigaciones en Ciencia y Tecnología de \\ Materiales - INTEMA, Consejo Nacional de Investigaciones Científicas y Técnicas - CONICET, \\ Universidad Nacional de Mar del Plata - UNMIP, Mar del Plata, Argentina \\ *mbagni@unsj.edu.ar
}

\begin{abstract}
Oily fractions of olive oil not suitable for human consumption used were: lamp oil (AL), olive pomace oil (OO) and clear oil lees (CB). Polyols were obtained by modifications of epoxidation with subsequent hydrolysis $(\mathrm{ALH}, \mathrm{OOH}, \mathrm{CBH})$ and transesterification (ALHT, OOHT, CBHT) in order to favor, then, the polymerization reactions. The analysis of the physicochemical properties determined show the decrease of the unsaturations in the triglyceride and the increase of the $\mathrm{OH}$ concentration in the modified polyols as compared to the initial oils (from 16 to 380 for the AL and the ALHT; from 24 to 448 for the $\mathrm{CB}$ and the $\mathrm{CBHT}$ and from 3 to 430 for the $\mathrm{OO}$ and the OOHT, respectively) .The main objective of this work is to evaluate the stability of these oils over time and to provide an alternative synthesis of polyurethanes from a renewable resource, not previously used for this purpose.
\end{abstract}

Keywords: biopolymers, oil stability, polyols, polyurethanes, synthesis.

How to cite: Bagni, M., Granados, D., \& Reboredo, M. (2019). Alternative use of oily fractions of olive oil. Polímeros: Ciência e Tecnologia, 29(1), e2019008. https://doi.org/10.1590/0104-1428.03618

\section{Introduction}

Polymeric materials from renewable resources have attracted a lot of attention in recent years. The development and utilization of vegetable oils for polymeric materials are currently in the spotlight of the polymer and chemical industry ${ }^{[1]}$. Vegetable oils have a relatively low cost, are abundant and widely available. They are triglycerides obtained by the esterification of glycerol and fatty acids (predominantly unsaturated fatty acids). These triglycerides have several active sites that can be the starting point for polymerization reactions giving rise to products of the polymer industry. Although they are heterogeneous in composition, the final properties of the polyurethanes will depend to a large extent on the content of the hydroxyls of the polyol (derived from triglycerides) and not of their composition $^{[2]}$.

The objective of this work is to develop green polyols from oily fractions derived from the olive industry not suitable for human consumption without previous refinement that can compete successfully with those polyols of petrochemical origin, in the area of polyurethanes. In addition, the stability of the oils during storage was studied in order to determine the potential existence of physicochemical changes that could later modify the polymerization reactions. When the oils are stored they can undergo two types of chemical modifications. One of them, the enzymatic rancidity (or hydrolytic rancidity), is produced by enzymes (lipases) found in both the embryo and the mesocarp of the fruit, responsible for converting triglycerides to fatty acids and glycerol, which results in an increase in the acidity of stored oils. These fatty acids show an increase until reaching a maximum from which they begin to descend. In turn, oxidative rancidity can occur, when the double bonds of the unsaturated fatty acids are oxidized in the presence of oxygen to peroxides or hydroperoxides that give rise to aldehydes, ketones, etc. Due to this, it is important to evaluate the oil physicochemical changes to predict the shelf life and in which stage of its storage it is convenient to use them as precursors of the polyols ${ }^{[3,4]}$.

The main contribution of this work is to provide an alternative synthesis of polymers from a renewable resource and propose a new option for the final disposal of these oily fractions, since no works have been found on polyurethanes or polyols from these wastes.

\section{Materials and Methods}

\subsection{Materia/s}

The oily raw materials used in this work were: Clear oil lees (CB), the oily stream extracted from the bottom of the decanters accompanied by sludges and vegetation water and that has begun to suffer oxidative processes; Lamp oil (AL), oily phase obtained from damaged or overripe olives and finally, Olive pomace oil (OO), residual oil extracted with solvents from the solid by-product generated during the process of olive oil extraction which is known as olive wet husk. All of these oily fractions need to be refined if they pretend to be suitable for human consumption. 
Lamp oil was obtained from Nucete Company, La Rioja, Argentina. Olive pomace oil was obtained from Olivsan, San Juan, Argentina, and Clear oil lees was obtained from 6 Marías Establishment, San Juan, Argentina. Hydrogen peroxide (30 wt $\%)$ from Anedra Laboratories and formic acid (85 wt $\%$ ) from Sintorgan Laboratories were used in the hydroxylation reaction. Triethanolamine (TEA $>99 \%$ ) from Biopack Laboratories and lithium hydroxide anhydrous (100\%) from Hach Company were used in the transesterification reaction.

In the preparation of the polyurethane polymers, the isocyanate used was a commercial polymeric MDI (4,4-diphenylmethane diisocyanate) Rubinate 1680 from Huntsman Polyurethanes, USA.

\subsection{Stability of oils}

The evolution with time of oils was studied by means of Fourier Transform Infrared Spectroscopy (FTIR) and by the determination of the Acid Value and the Iodine Index. The FTIR spectra were obtained using a Nicolet 6700 spectrometer from Thermo Scientific in absorbance mode, operated at a resolution of $2 \mathrm{~cm}^{-1}$, in a complete working range of 4000-500 $\mathrm{cm}^{-1}$. Acid value measurement was performed according to the Free Fatty Acids Method, AOCS Official Method Ca 5a-40. Iodine Index was determined by the Wijs method American Oil Chemists' Society, AOCS Official Method Cd 1-25. The spectra found by Fourier Transform Infrared Spectroscopy were performed on samples without storage time, with one year and three years of storage, while the acid value and the iodine index tests were performed on oils without storage time and on oils with 2,2 and a half and 3 years of storage and on oils without storage time and on oils with 1, 2 and 3 years of storage, respectively.

\subsection{Synthesis of polyols}

Epoxidation with subsequent hydrolysis (Hydroxylation): Hydrogen peroxide together with formic acid were added to a stirred reactor at a temperature of $40{ }^{\circ} \mathrm{C}$. The initial ratio used was $\mathrm{H}_{2} \mathrm{O}_{2} / \mathrm{HCOOH}$ (weight) $=1 / 1.9$. The oil was added dropwise with an initial ratio oil/ $/ \mathrm{H}_{2} \mathrm{O}_{2}$ (weight) of $3.5 / 1$ and the temperature was maintained between 40 and $50{ }^{\circ} \mathrm{C}$ for 3 hours. Then the final product was cooled to room temperature. The liquid separated clearly into two phases. The upper phase was recovered. During this stage, hydrogen peroxide reacts with formic acid to form performic acid. Once the oil is added, it goes through an intermediate stage of epoxidation. The epoxy ring is unstable under acidic conditions so it opens up giving rise to the formation of hydroxyl groups $\mathrm{s}^{[5]}$.

Transesterification of the hydroxylated oil: The hydroxylated oil, as well as that obtained in the epoxidation stage, (ALH, $\mathrm{OOH}$ and $\mathrm{CBH}$ ), triethanolamine and lithium hydroxide were placed together in a reactor with mechanical agitation. The temperature was raised to $150^{\circ} \mathrm{C}$ in 30 minutes and then maintained at this value for 2.5 hours. This is a complex stage consisting in the transesterification of the triglyceride molecules with triethanolamine to give rise to a mixture of different polyalcohols containing one or two chains of hydroxylated fatty acids (ALHT, OOHT, CBHT). No further purification steps were performed on the obtained polyol.
The physicochemical characterization of the oils and their modifications were carried out through measurements of viscosity, density, iodine value, hydroxyl value, water content and volatile material, acid value, saponification index, Fourier Transform Infrared Spectroscopy (FTIR) and Nuclear Magnetic Resonance (NMR). The FTIR spectra were obtained under the same conditions used to determine the stability of the oils.

The NMR measurements were made using a spectrometer brand BRUKER model AVANCE II. It operates at a frequency of $300.13 \mathrm{MHz}$ for protons. A probe of CP/MAS was used with rotors for HRMAS of $4 \mathrm{~mm}$ in diameter and volume of $57 \mu \mathrm{L}$. The protons spectra were recorded using the technique of direct polarization and rotation to the magic angle. The pulse of proton excitation $(\pi / 2)$ was $3.8 \mu \mathrm{s}$ of duration, the repetition time was $3 \mathrm{~s}$ and the speed of rotation of the sample $3 \mathrm{kHz}$. There were 32 averages per spectrum. The samples were tested as received without any extra treatment.

\subsection{Synthesis of polyurethane}

Synthesis tests were carried out by reacting the obtained polyols (ALHT, CBHT, OOHT) with diisocyanate. The molar ratio used was $\mathrm{NCO} / \mathrm{OH}=1.1$. The system was manually stirred for 30 seconds and the temperature was recorded during the test.

\section{Results and Discussions}

\subsection{Stability of oils}

Figure 1 shows the infrared spectra of the $\mathrm{CB}$ oils according to the passage of time (only one of the fractions is shown since the spectra for the three oils are similar). The main chemical change suffered by the oils during storage was the increase of the acid groups as a consequence of the enzymatic hydrolysis, responsible for breaking down triglycerides into free fatty acids and glycerol (enzymatic rancidity). In the spectra of $\mathrm{CB}$ and $\mathrm{OO}$ the appearance of a small peak at $1710 \mathrm{~cm}^{-1}$ (acidic groups) adjacent to the ester peak $\left(1750 \mathrm{~cm}^{-1}\right)$ in the sample stored for 3 years was detected, which in oils without storage was imperceptible. In the AL sample it was observed that the peak corresponding to the acid group conserved the same size. Table 1 shows the results of acid value for the three samples analyzed in different periods of time studied. Total correspondence with the information provided by the FTIR spectra can be observed.

On the other hand, it could be affirmed that the samples have suffered oxidative rancidity due to the presence of oxygen during storage. Oxygen is responsible for oxidizing the double bonds present in the fatty acids, giving rise to the formation of peroxides or hydroperoxides. Table 2 shows the decrease in iodine values during storage, which can directly translate into a decrease in unsaturation.

Therefore, it can be concluded that it is convenient to carry out the hydroxylation and transesterification reactions to obtain polyols to samples without storage time, in order to have the highest number of double bonds in the samples prone to hydroxylation. 


\subsection{Characterization of oils and their modifications}

The main differences in the FTIR spectra between untreated oils versus the hydroxylated and transesterified samples (OO versus OOHT) can be seen in Figure 2

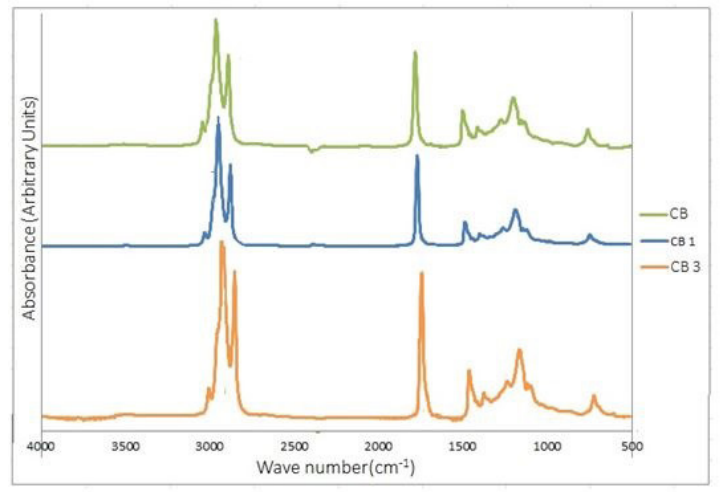

a

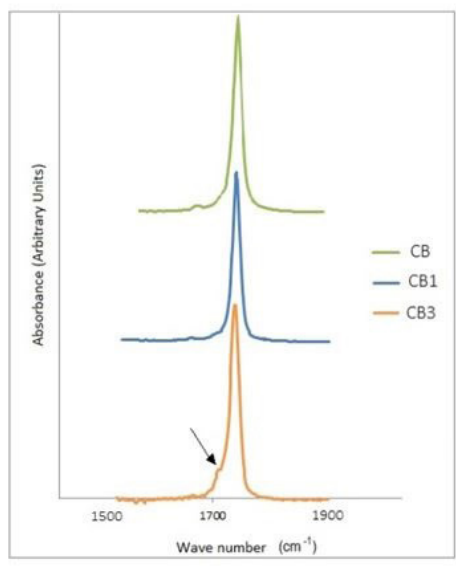

b

Figure 1. (a) FTIR spectra of clear oil lees at different periods of storage. $\mathrm{CB}, \mathrm{CB} 1$ and $\mathrm{CB} 3$, without storage, with 1 year and 3 years of storage, respectively; (b) Peak at $1750 \mathrm{~cm}^{-1}$ corresponding to the ester group and a peak at $1710 \mathrm{~cm}^{-1}$ corresponding to acid groups in the clear oil lees. (only one of the oily fractions is shown since the spectra are similar) and lie in the appearance of a broad peak to $3400 \mathrm{~cm}^{-1}$ corresponding to the $\mathrm{OH}$ groups incorporated in the treated samples at the expense of the decrease of the peak corresponding to the unsaturations present at $3010 \mathrm{~cm}^{-1[6]}$. Table 3 shows the increase in $\mathrm{OH}$ values experimentally found by analytical techniques ${ }^{[7]}$. These values agree with the information provided by the spectra of each sample.

Similar behavior has been observed in tung oil samples ${ }^{[5]}$. The stretching vibration at $1746 \mathrm{~cm}^{-1}$ is attributed to the ester carbonyl funcional group of the triglycerides. The existence of peaks at 965 and $735 \mathrm{~cm}^{-1}$ indicates that both cis and trans conformations are present in the fatty acids that form the triglyceride ${ }^{[6]}$.

In turn, NMR spectra corroborate these chemical modifications (Figures 3, 4 and 5). In these, the presence of new resonances in the hydroxylated and transesterified samples can be observed. They appear in the range 5.09-5.29 ppm and are assigned to protons of $\mathrm{OH}$ groups. The signal of the unsaturated groups is still present (at $5.40 \mathrm{ppm}$ ) but decreased by less than half in intensity with respect to the unmodified sample. The signal of the protons of the $\mathrm{CH}_{2}$ groups neighboring to the unsaturated groups is also diminished (also in the middle), which is consistent with the decrease of unsaturated groups ${ }^{[5]}$. In the AL sample at 8 ppm (Figure $3 \mathrm{~b}$ ), a broad signal appears which normally corresponds to protons of carboxylic acid groups and which is not present in the $\mathrm{CB}$ and $\mathrm{OO}$ samples, since the lamp oil has the highest acidity index (see Table 1). The two

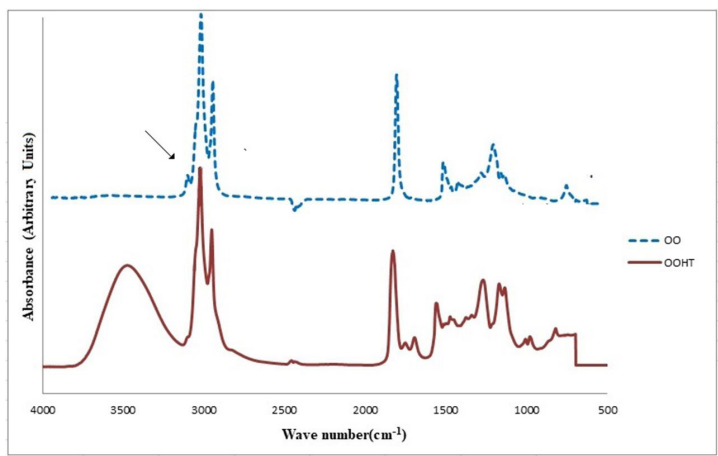

Figure 2. FTIR spectra of olive pomace oil and hydroxylated and transesterified olive pomace oil.

Table 1. Comparison of the acidity index of oils.

\begin{tabular}{ccccc}
\hline \multicolumn{5}{c}{ Acidity Index \% } \\
\hline Samples & without storage & 2 years of storage & 2 $1 / 2$ years of storage & 3 years of storage \\
\hline AL & $9.7 \pm 0.1$ & $10.7 \pm 0.3$ & $10.5 \pm 0.1$ & $10.4 \pm 0.3$ \\
CB & $1.1 \pm 0.2$ & $1.0 \pm 0.1$ & $5.2 \pm 0.1$ & $5.6 \pm 0.2$ \\
OO & $2.0 \pm 0.1$ & $1.6 \pm 0.4$ & $2.5 \pm 0.1$ & $2.7 \pm 0.2$ \\
\hline
\end{tabular}

Table 2. Iodine index for different storage times.

\begin{tabular}{ccccc}
\hline \multicolumn{5}{c}{ Iodine Index (Wijs) } \\
\hline Samples & without storage & 1 years of storage & 2 years of storage & 3 years of storage \\
\hline $\mathrm{AL}$ & $\mathbf{8 0} \pm \mathbf{2}$ & $\mathbf{8 3} \pm \mathbf{2}$ & $\mathbf{7 7 \pm 2}$ & $\mathbf{7 6} \pm \mathbf{7}$ \\
$\mathrm{CB}$ & $\mathbf{8 4} \pm \mathbf{1}$ & $\mathbf{8 4} \pm \mathbf{1}$ & $\mathbf{7 9} \pm \mathbf{3}$ & $\mathbf{6 9} \pm \mathbf{8}$ \\
$\mathrm{OO}$ & $\mathbf{8 3} \pm \mathbf{1}$ & $\mathbf{8 1} \pm \mathbf{3}$ & $\mathbf{7 5} \pm \mathbf{2}$ & $\mathbf{7 4 \pm 9}$ \\
\hline
\end{tabular}


Table 3. $\mathrm{OH}$ value for oils and their modifications.

\begin{tabular}{ccccccc}
\hline Samples & AL & ALHT & CB & CBHT & OO & OOHT \\
\hline Value $\mathrm{OH} \mathrm{mgKOH} / \mathrm{g}$ & $\mathbf{1 6}$ & $\mathbf{3 8 0}$ & $\mathbf{2 4}$ & $\mathbf{4 4 8}$ & $\mathbf{3}$ & $\mathbf{4 3 0}$ \\
\hline
\end{tabular}

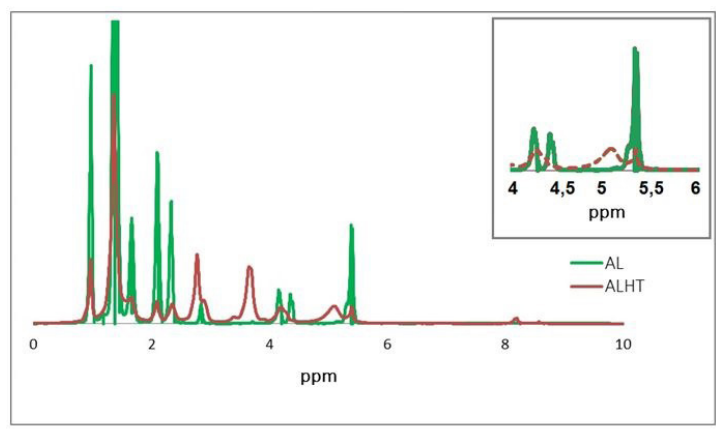

a

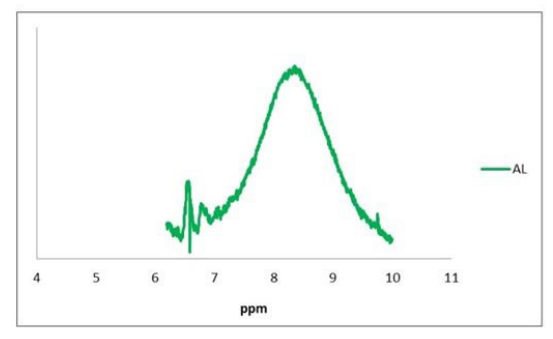

b

Figure 3. (a) NMR spectra of lamp oil and hydroxylated and transesterified lamp oil; (b) Resonance present at $8 \mathrm{ppm}$ in AL, corresponding to acid groups.

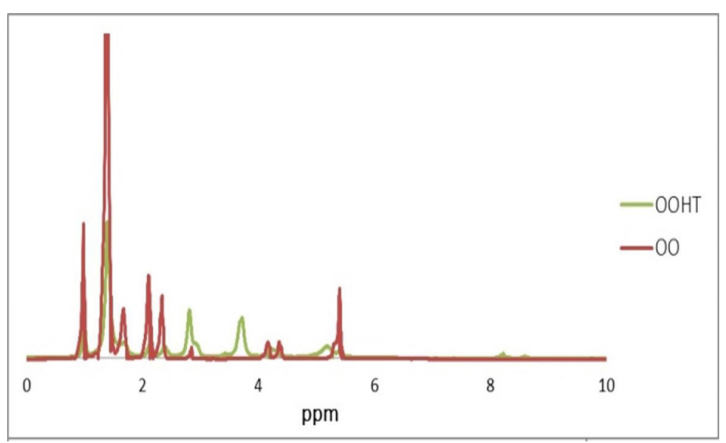

Figure 4. NMR spectra of olive pomace oil and hydroxylated and transesterified olive pomace oil.

multiplets at 4.4 and $4.1 \mathrm{ppm}$ corresponds to the four glycerol methylene protons in the triglyceride molecule, while the small multiplet at $5.3 \mathrm{ppm}$ corresponds to the central $\mathrm{H}$ in the glycerol moieties.

The physicochemical characterization determined that after the modification reactions (epoxidation with subsequent hydrolysis and transesterification) an increase of hydroxyl groups (greater number of hydroxyls) was reached, which is reflected in an increase in viscosity and reduction of

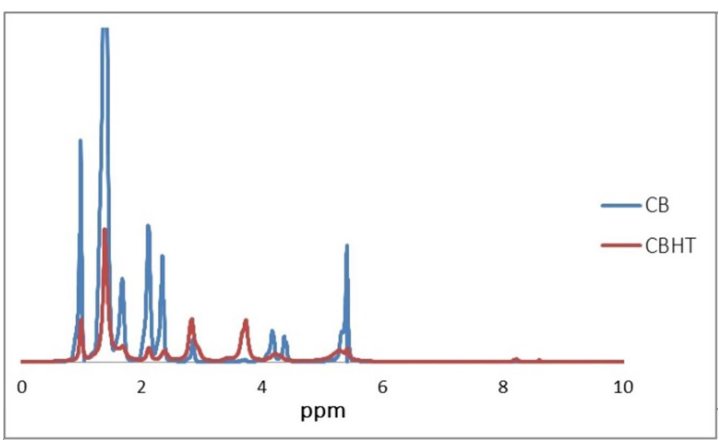

Figure 5. NMR spectra of clear oil lees and hydroxylated and transesterified clear oil lees.

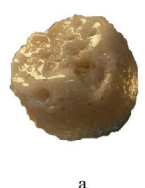

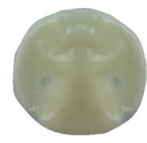

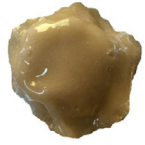

c
Figure 6. Polyurethane obtained from OOHT (a), from CBHT (b) and from ALHT (c).

iodine index due to the opening of fatty acid unsaturations to achieve their incorporation ${ }^{[7]}$.

\subsection{Synthesis of polyurethanes}

Preliminary tests of polyurethane synthesis were performed by the reaction of the obtained polyols (ALHT, CBHT, OOHT) with diisocyanate (RUBINATE 1680), as it can be seen in Figure 6.

The system was manually stirred for 30 seconds. Opalescence, foaming and gelation times and the reaction temperature were recorded throughout the test (see Table 4). The polymerization reaction between the OOHT polyol with the diisocyanate is the most exothermic and has the shortest opalescence, foaming and gelation times. The curing reaction occurs faster and its final appearance is porous, which could be attributed to the water content remaining in the oil. The final volume is more than twice the initial volume for the ALHT and OOHT samples while the CBHT only increases by $66 \%$.

The reactions between the ALHT and CBHT polyols with diisocyanate present similar opalescence, foaming and gelation times. The main difference found between these samples is their final appearance. The sample obtained from ALHT is more compact, while the sample obtained from CBHT has a greater number of small pores. The samples are in the characterization stage, which includes tests of FTIR, DSC (differential scanning calorimetry), TGA (thermogravimetry), among others. 
Table 4. Characteristics observed during the synthesis of polyurethane.

\begin{tabular}{|c|c|c|c|c|c|c|c|}
\hline Samples & $\begin{array}{c}\text { Initial } \\
\text { Volume }(\mathrm{mL})\end{array}$ & $\begin{array}{c}\text { Final Volume } \\
(\mathrm{mL})\end{array}$ & $\begin{array}{c}\text { Opalescence } \\
\text { (s) }\end{array}$ & $\begin{array}{c}\text { Temperature } \\
\left({ }^{\circ} \mathrm{C}\right) \\
4 \mathrm{~min} \\
\end{array}$ & $\begin{array}{c}\text { Foaming } \\
\text { time (s) }\end{array}$ & $\begin{array}{l}\text { Gelling } \\
\text { time (s) }\end{array}$ & $\begin{array}{c}\text { Final } \\
\text { Appearance } \\
(3 \mathrm{~h}) \\
\end{array}$ \\
\hline ALHT & 12 & 25 & 10 & 48 & 112 & 180 & Rubbery \\
\hline CBHT & 12 & 20 & 20 & 48 & 120 & 180 & Pasty \\
\hline OOHT & 12 & 25 & 5 & 58 & 90 & 120 & Rigid \\
\hline
\end{tabular}

\section{Conclusions}

The study of the stability of the oils allowed to know in which stage of the storage it is convenient to chemically modify the oils to obtain the polyols. Therefore, it can be concluded that the oils must be treated without storage time since it is when they present a greater amount of unsaturations that can be hydroxylated and thus contribute to the increase of the content of $\mathrm{OH}$ groups in the polyols.

By means of epoxidation and transesterification reactions, it was possible to increase the content of $\mathrm{OH}$ groups in order to obtain polyols that give rise to thermoset polyurethanes. The three oily fractions adopted are suitable for carrying out polymerization reactions with diisocyanates.

The three polymeric materials obtained present well differentiated aspects. Subsequent tests of physicochemical characterization will allow to associate the resulting properties of the polymers with the characteristics of the chemically modified oily fractions.

\section{Acknowledgements}

The authors gratefully acknowledge the CONICET for the doctoral fellowship of M.M. Bagni and for the financial support, as well as Mr. Oscar Casemayor and Dr. Diana Fasce for the chemical and analytical measurements.

\section{References}

1. Samarth, N. B., \& Mahanwar, P. A. (2015). Modified vegetable oil based additives as a future polymeric material. Open
Journal of Organic Polymer Materials, 5(1), 1-22. http:// dx.doi.org/10.4236/ojopm.2015.51001.

2. Petrović, Z. S., Zhang, W., Zlatanić, A., Lava, C. C., \& Ilavskyý, M. (2002). Effect of OH/NCO molar ratio on properties of soy-based polyurethane networks. Journal of Polymers and the Environment, 10(1-2), 5-12. http://dx.doi. org/10.1023/A:1021009821007.

3. Martinez C. (2018). Determination of the acidity index in oils and edible fats. Retrieved in 2018, May 28, from https:// es.scribd.com/doc/97574878/Determinacion-del-indice-deacidez-en-aceites-y-grasas-comestibles

4. Shahidi, F., \& John, J. A. (2013). Oxidative rancidity in nuts. In L. Harris (Ed.), Improving the safety and quality of nuts (pp. 198-229). Cambridge: Woodhead Publishing. http://dx.doi. org/10.1533/9780857097484.2.198

5. Vlachos, N., Skopelitis, Y., Psaroudaki, M., Konstantinidou, V., Chatzilazarou, A., \& Tegou, E. (2006). Applications of Fourier transform-infrared spectroscopy to edible oils. Analytica Chimica Acta, 573-574, 459-465. http://dx.doi.org/10.1016/j. aca.2006.05.034. PMid:17723561.

6. Bagni, M., Reboredo, M., \& Granados, D. (2015). Use of oily wastes to obtain polymers. In Latin American Congress of Engineering and Applied Sciences (p. 201). Mendoza: National University of Cuyo.

7. Mosiewicki, M., Casado, U., Marcovich, N., \& Aranguren, M. (2009). Polyurethanes from tung oil: polymer characterization and composites. Polymer Engineering and Science, 49(4), 685-692. http://dx.doi.org/10.1002/pen.21300.

Received: May 29, 2018

Revised: July 11, 2018

Accepted: July 17, 2018 